\title{
Twice-cooperative Spectrum Sensing Based on Double Threshold Energy Detection
}

\author{
Bingmei Miao a, Yafang Wang ${ }^{b}$, Yan $\mathrm{Li}^{\mathrm{c}}$ and Chang Cai ${ }^{\mathrm{d}}$ \\ Hebei University of Science and Technology, Shijiazhuang 050000, China. \\ a2300692047@qq.com, b1329828875@qq.coml, c1039544879@qq.com, d2396609949@qq.com
}

Keywords: cognitive radio network, twice-cooperative spectrum sensing, double threshold, energy detection, hard decision fusion.

\begin{abstract}
Double threshold energy detection based cooperative spectrum sensing exist the problem of perceived failure and need larger bandwidth to transmit cognitive users' perceive information. In this paper, we propose a method for two problems of spectrum sensing in cognitive radio networks (CRNs) aiming to increase the detection performance of the spectrum sensing. The method can dynamically change the threshold value according to the perceived result of the previous time, and then determine the existence state of the primary user when the energy value is between the high and low threshold values. Twice-cooperative spectrum sensing based on double threshold energy detection can reduce the perceive information transferred to the fusion decision center and save the transmission bandwidth. The simulation results show that this method can effectively improve the detection probability of cooperative spectral sensing and reduce the probability of missed detection.
\end{abstract}

\section{Introduction}

With the rapid development of wireless communication technology, the shortage of spectrum resources is becoming more and more serious [1]. The main reason for this situation is that the spectrum resources are not fully utilized due to the fixed spectrum management policies. In order to solve the problem, the concept of cognitive radio (CR) is proposed by Mitola et al [2]. The purpose is hoping cognitive users can access to the authorized spectrum of the idle state. The communication quality of primary users must be ensured during the data transfer process. So cognitive users have to be able to accurately and quickly perceive the spectrum around them, and determine the state of primary users, which is spectrum sensing technology. Cooperative spectrum sensing technology can solve the problem of low signal-to-noise ratio (SNR), shadow effect, multipath fading to some extent $[3,4]$, so it becomes the most widely used spectrum sensing technology [5].

Among the local detection technology for cooperative spectrum sensing, energy detection implementation is simple, do not need to know the primary users of priori information, and did not limit detection signal types, high practicality, therefore is most used in cooperative spectrum sensing local detection technology [6]. Traditional energy detection algorithm sampled samples is big, in the low SNR situation detection performance is poorer, therefore this paper proposes a small sample of double threshold cooperative spectrum sensing method, this method effectively reduces the cognitive user's interference with the primary user, but when the energy value is between the high and low threshold values exist perceive failure [7]. To solve the problem of perceive of failure in double threshold energy detection, of cooperative spectrum sensing is put forward to the local ruling divided into perceive information between the high and low threshold and local decision two parts, the use of decision fusion center for soft and hard decision of this respectively, and then make a final decision, to determine the state of primary users, this method has solved the perception of failure problem in double threshold energy detection, but increased the computational complexity of decision fusion center [8]. In this paper, a twice-cooperative spectrum sensing method based on double threshold energy detection is proposed and analyzes the system detection performance under Additive White Gaussian Noise (AWGN) channel. 


\section{Traditional cooperative spectrum sensing}

The principle of energy detection is by comparing the energy value and the threshold set in advance. If the energy value is less than the threshold, then the primary user does not exist; otherwise there is the primary user. The spectrum sensing model can be represented as a binary hypothesis model based on the occupancy of the primary user's signal. Set $v(k)$ to be AWGN signal, $x(k)$ indicates the signal received by the cognitive user, $s(k)$ is the transmitter signal of the primary user, the binary hypothesis model can be expressed as:

$$
\begin{cases}H_{0}: x(k)=v(k), & k=1,2, \ldots, K \\ H_{1}: x(k)=s(k)+v(k) & k=1,2, \ldots, K\end{cases}
$$

Where $H_{0}$ indicates that the primary user signal is not present in the detected frequency band, and $H_{1}$ indicates that the primary user signal exists in the detected frequency band. Using the method of mathematical statistics, according to the energy value accumulated by $x(k)$, the judgment statistic is defined as:

$$
Y=\sum_{k=1}^{K} x^{2}(k)
$$

The judgment statistic follows the chi-square distribution, that is:

$$
Y= \begin{cases}x_{2 u}^{2}(2 \gamma), & H_{0} \\ x_{2 u}^{2}, & H_{1}\end{cases}
$$

Where $u$ is the time domain bandwidth product proportional to the sample number $\mathrm{K}, x_{2 u}^{2}(2 \gamma)$ is a noncentral chi-square distribution with a degree of freedom of $2 u, x_{2 u}^{2}$ is a center chi-square distributio with a degree of freedom of $2 u, \gamma$ is the SNR. Under AWGN channel, set the low threshold value as $E_{t h 1}$ and the high threshold value as $E_{t h 2}$.

In double threshold energy detection, if the energy value $Y>E_{t h 2}$, the perceptual result is $H_{1}$, which indicates the primary user signal is in the detection frequency band, and the cognitive user can not use the frequency band. If the energy value $Y<E_{t h 1}$, the perceptual result is $H_{0}$, which indicates the primary user signal is not in the detection frequency band, and the cognitive user can use the frequency band. If the received energy value is between the high and low threshold values ( $E_{t h 1}<Y<E_{t h 2}$ ), it does not enter this decision and perform spectrum sensing again. The formulas for detection probability $P_{d}$, false alarm probability $P_{f}$, and missed detection probability $P_{m}$ of double threshold energy detection under AWGN channel are:

$$
\begin{aligned}
& P_{d}=P\left(Y \geq E_{t h 2} \mid H_{1}\right)=Q_{u}\left(\sqrt{2 \gamma}, \sqrt{E_{t h 2}}\right) \\
& P_{f}=P\left(Y \geq E_{t h 2} \mid H_{0}\right)=\frac{\Gamma\left(u, E_{t h 2} / 2\right)}{\Gamma(u)} \\
& P_{m}=P\left(Y<E_{t h 1} \mid H_{1}\right)=1-Q_{u}\left(\sqrt{2 \gamma}, \sqrt{E_{t h 1}}\right)
\end{aligned}
$$

The detection probability $P_{d}$ represents the probability of cognitive users correctly detects primary user signal presence in the test band. The false alarm probability $P_{f}$ indicates that there is no primary user in the test band, but the $Y$ value is greater than the threshold value. In other words, the energy detector thinks there is a primary user. Missed detection probability $P_{m}$ indicates that there is a primary user in the test band, but the $Y$ value is less than the threshold value. In other words, the energy detector does not think there is a primary user.

In the formula $Q_{u}(a, b)$ is the Generalized Marcum Q function, $Q_{u}(a, b)=\frac{1}{a^{u-1}} \int_{b}^{+\infty} t^{u} e^{\frac{t^{2}+a^{2}}{2}} I_{u-1}(a t) d t$. Where $I_{u-1}(\bullet)$ represents the first type of modified Bessel function, u-1 order. $\Gamma(a, b), \Gamma(a)$ denote the incomplete and complete Gamma functions, where $\Gamma(a, b)=\int_{b}^{+\infty} t^{a-1} e^{-t} d t$. 
Traditional cooperative spectrum sensing is cognitive users using the double threshold energy detection perceive the same frequency band, transfer perceive information to the fusion decision center, and then according to the decision fusion determine the state of the primary user. The flow chart diagram is shown in figure1.

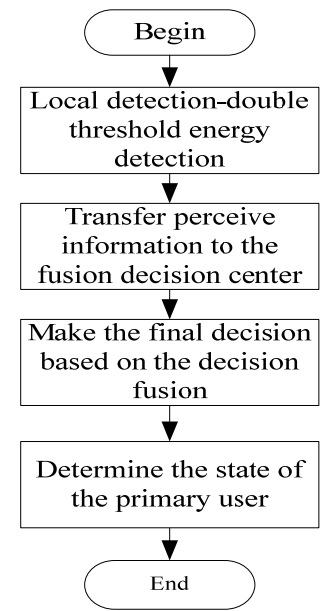

Fig. 1 Traditional cooperative spectrum sensing flow chart

\section{Twice-cooperative Spectrum Sensing}

Although the traditional cooperative spectrum sensing improves the detection performance, but there is a third state, means the value is $E_{t h 1}<Y<E_{t h 2}$, which judgment result is not obtained, and reduce the reliability of the detection performance. At present, the solution to this problem is mainly through performing spectrum sensing multiple times, but this solution increases the time of spectrum sensing. Therefore put forward the method of the twice-cooperative spectrum sensing based on improved double threshold energy detection. That is, the third state between in high and low threshold is improved and through a spectrum of perception can determine the existence of the primary user status, which shorten the perception time. The flow chart diagram of improved double threshold energy detection is shown in figure 2.

The formulas for detection probability $P_{d}$, false alarm probability $P_{f}$, and missed detection probability $P_{m}$ of improved double threshold energy detection under AWGN channel are:

$$
\begin{aligned}
& P_{d}=P\left(H_{0}\right) \times Q_{u}\left(\sqrt{2 \gamma}, \sqrt{E_{t h 2}}\right)+P\left(H_{1}\right) \times Q_{u}\left(\sqrt{2 \gamma}, \sqrt{E_{t h 1}}\right) \\
& P_{f}=P\left(H_{0}\right) \times \frac{\Gamma\left(u, E_{t h 2} / 2\right)}{\Gamma(u)}+P\left(H_{1}\right) \times \frac{\Gamma\left(u, E_{t h 1} / 2\right)}{\Gamma(u)} \\
& P_{m}=P\left(H_{0}\right) \times\left[1-Q_{u}\left(\sqrt{2 \gamma}, \sqrt{E_{t h 2}}\right)\right]+P\left(H_{1}\right) \times\left[1-Q_{u}\left(\sqrt{2 \gamma}, \sqrt{E_{t h 1}}\right)\right]
\end{aligned}
$$

Among:

$P\left(\mathrm{H}_{0}\right)+P\left(\mathrm{H}_{1}\right)=1$

Set the detection probability of the traditional double threshold energy detection and improved double threshold energy detection is $P_{d 1}$ and $P_{d 2}$, and the missed detection probability is $P_{m 1}$ and $P_{m 2}$, and compare the magnitude of $P_{d 1}$ and $P_{d 2}, P_{m 1}$ and $P_{m 2}$.

$$
\begin{aligned}
& P_{d 2}-P_{d 1}=P\left(H_{0}\right) \times Q_{u}\left(\sqrt{2 \gamma}, \sqrt{E_{t h 2}}\right)+P\left(H_{1}\right) \times Q_{u}\left(\sqrt{2 \gamma}, \sqrt{E_{t h 1}}\right)-Q_{u}\left(\sqrt{2 \gamma}, \sqrt{E_{t h 2}}\right. \\
& >\left[P\left(H_{0}\right)+P\left(H_{1}\right)\right] \times P\left(\mathrm{Y} \geq \mathrm{E}_{\mathrm{th} 2} \mid \mathrm{H}_{1}\right)-P\left(\mathrm{Y} \geq \mathrm{E}_{\mathrm{th} 2} \mid \mathrm{H}_{1}\right)=0
\end{aligned}
$$

$P_{d 2}>P_{d 1}$ Indicates the improved double threshold energy detection increases the detection probability of the system. Similarly, $P_{m 2}<P_{m 1}$ can be proved, which indicates this method reduces the missed detection probability of the system. 


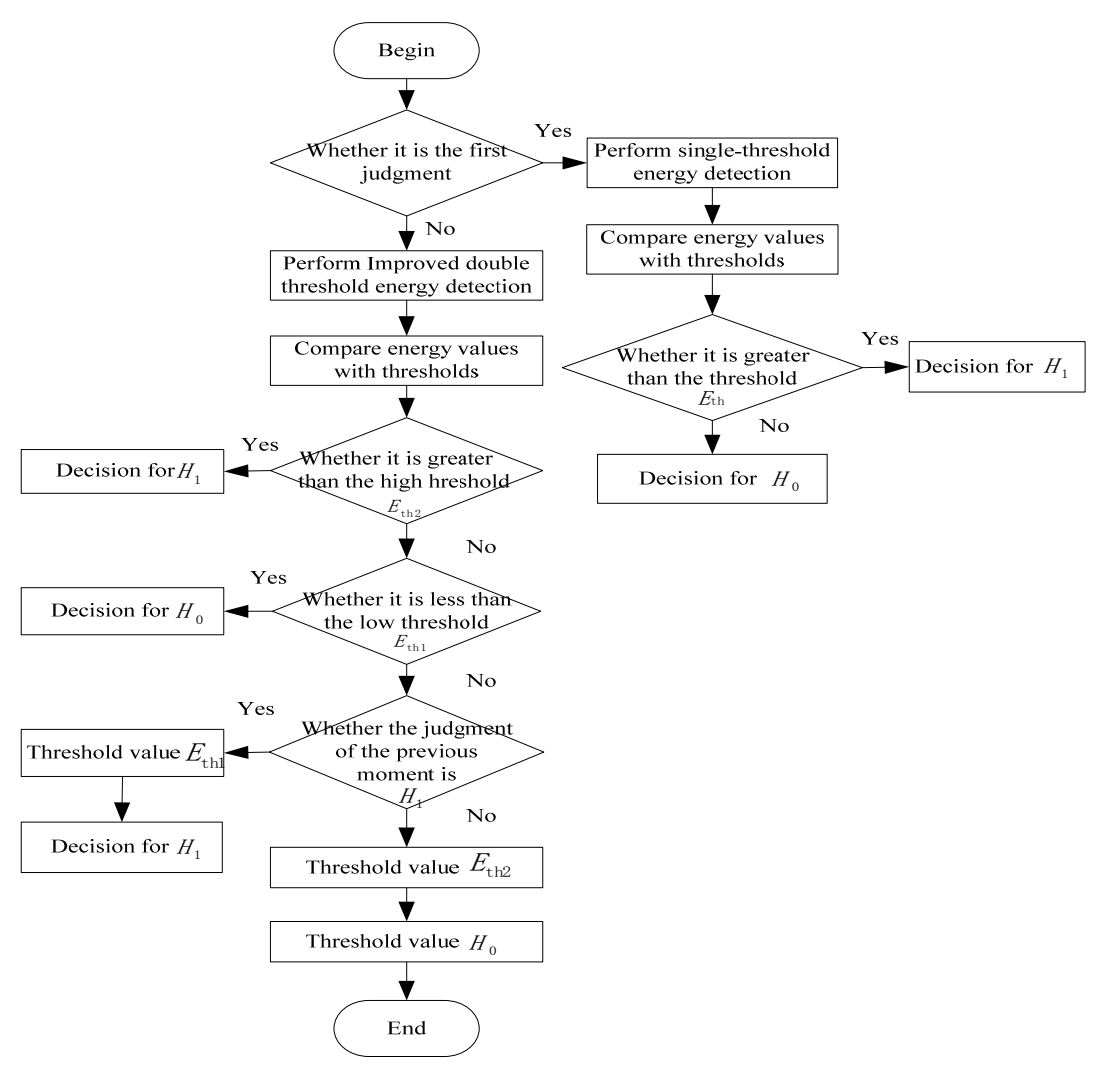

Fig. 2 Improved double threshold energy detection flow chart

In the process of twice-cooperative spectrum sensing based on improved double threshold energy detection, we assume that cognitive users are divided into different groups by upper layers, each group is independent of each other [9], and the cognitive users in the group are also independent. In each group, the cognitive radio can choose a cognitive user with the maximum SNR as the cluster-head, which collects the local decision from other cognitive users, and conducts the group internal decision fusion then sends the fusion result to cognitive base (first cooperation). Cognitive base receives the fusion result from cluster-head, and makes the final decision using decision fusion. Determine the state of the primary user (second cooperation). The flow chart diagram of twice-cooperative spectrum sensing is shown in figure 3.

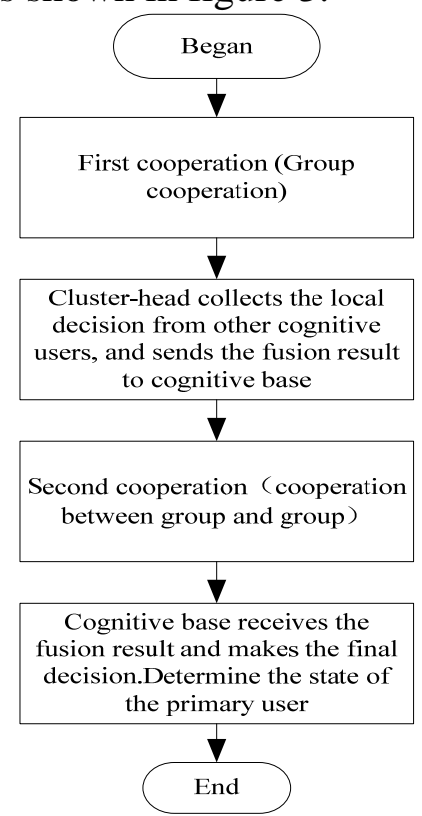

Fig. 3 Twice-cooperative spectrum sensing flow chart 


\section{Simulation and Analysis}

In this section, we use MATLAB to simulate the spectrum sensing under "OR" logic operation and "AND" logic operation. The simulation results are presented by the cooperative spectrum sensing ROC (complementary receiver operating characteristic) curve. Simulation of transmission channel selecting AWGN channel. The number of cognitive users involved in the cooperative is 10.The number of groups is 2 and the number of every group is 4 and 6, the SNR of cooperative users submit to the range of $(-18,-19) \mathrm{dB}$ of uniform distribution. When given the decision fusion criteria and the fusion decision false alarm probability, the false alarm probability of each cognitive user can be obtained by the relationship between the false alarm probability of fusion decision and the false alarm probability of each cognitive user [10], then determine the decision threshold value as $E_{t h}$ [4].Set the low threshold value as $E_{t h 1}=0.8 * E_{t h}$, the high threshold value as $E_{t h 2}=1.2 * E_{t h}$, and the time domain bandwidth product $u=5$.
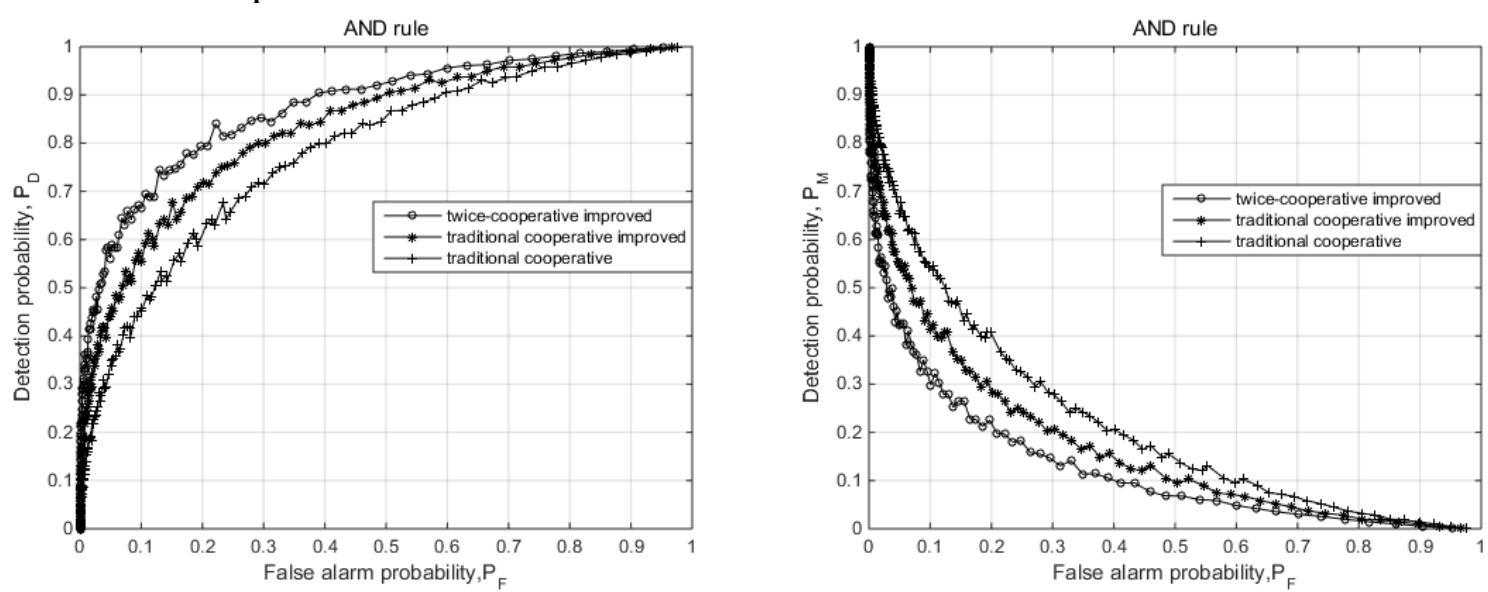

Fig. 4 (a) Detection probability comparison (b) Missed detection probability comparison

The ROC curve of the three plans is shown in figure 4 when the decision fusion criterion is the “AND” logic operation. Compared with traditional cooperative spectrum sensing based on improved double threshold energy detection, the detection probability of twice-cooperative spectrum sensing based on improved double threshold energy detection can be increased by about $12 \%$, the missed detection probability can be reduced by about $25 \%$. Compared with traditional cooperative spectrum sensing based on double threshold energy detection, the detection probability of traditional cooperative spectrum sensing based on improved double threshold energy detection can be increased by about $11 \%$, the missed detection probability can be reduced by about $25 \%$.

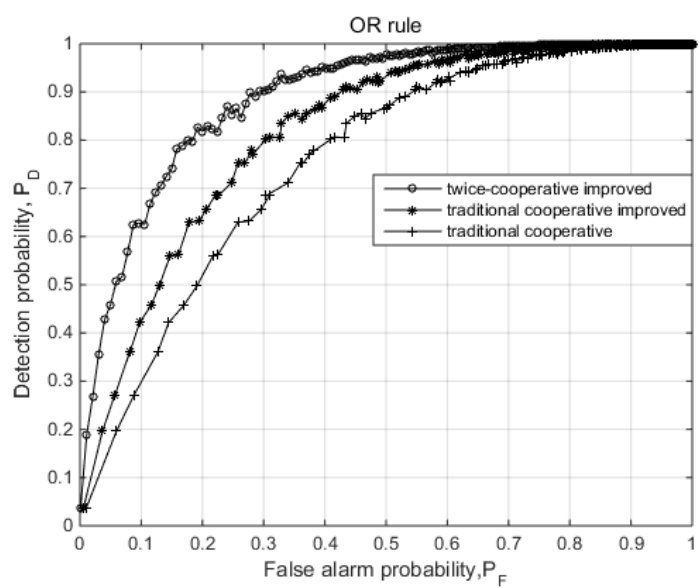

Fig. 5 (a) Detection probability comparison

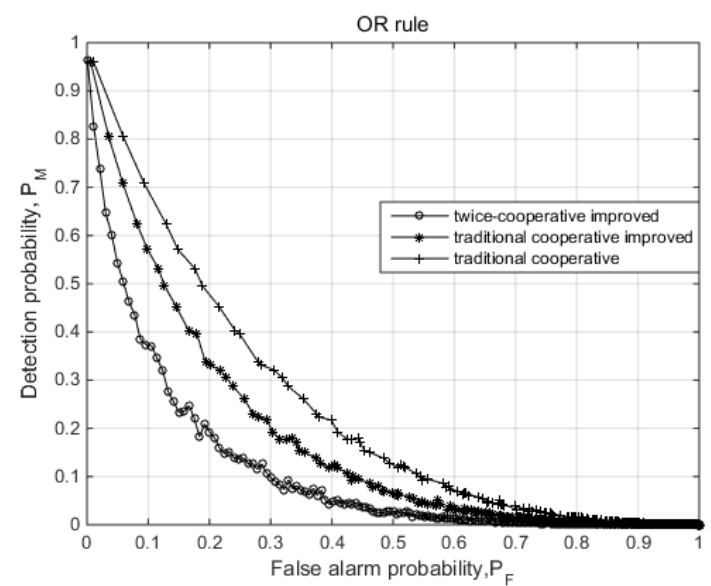

(b) Missed detection probability comparison

The ROC curve of the three plans is shown in figure 5 when the decision fusion criterion is the "OR" logic operation. Compared with traditional cooperative spectrum sensing based on improved double threshold energy detection, the detection probability of twice-cooperative spectrum sensing based on improved double threshold energy detection can be increased by about $24 \%$, the missed detection probability can be reduced by about 30\%. Compared with traditional cooperative spectrum 
sensing based on double threshold energy detection, the detection probability of traditional cooperative spectrum sensing based on improved double threshold energy detection can be increased by about $15 \%$, the missed detection probability can be reduced by about $30 \%$.

\section{Conclusion}

Through the comparison of twice-cooperative spectrum sensing based on improved double threshold energy detection, traditional cooperative spectrum sensing based on improved double threshold energy detection and traditional cooperative spectrum sensing based on double threshold energy detection, the detection performance of twice-cooperative spectrum sensing based on improved double threshold energy detection is better.

The ROC curves for "AND" and "OR" rules in decision fusion strategy, which detection probability can be increased by about $12 \%$ and $24 \%$ compared with traditional cooperative spectrum sensing. The missed detection probability can be reduced by about $25 \%$ and $30 \%$ compared with traditional cooperative spectrum sensing. Not only improves the systematic detection performance, also reduces the amount of computing in the fusion decision center, saving bandwidth.

\section{References}

[1]. Stanislav Filin, Hiroshi Harada, Homare Murakami, et al. International Standardization of Cognitive Radio Systems. IEEE Communications Magazine. Vol. 49 (2011) No. 3, p. 82-89.

[2]. Erik Axell, Geert Leus, Erik G. Larsson, et al. Spectrum Sensing for Cognitive Radio: State-of-the-Art and Recent Advances. IEEE Signal Processing Magazine. Vol. 29 (2012) No. 3, p. 101-106.

[3]. Chen Yonghong, Guo Lili, Zhang Shibing. Cooperative Spectrum Sensing and Performance Analysis under Fading Channel. Communications Technology. Vol. 47 (2014) No. 9, p. 1031-1036.

[4]. Eleftherios Chatziantoniou, Ben Allen, and Vladan Velisavljevic. Threshold Optimization for Energy Detection-Based Spectrum Sensing Over Hyper-Rayleigh Fading Channels. IEEE Communications Letters. Vol. 19 (2015) No. 6, p. 1077-1080.

[5]. Zhu Jiang, Huang Ben-xiong, Wang Fu-rong, et al. Novel Method of Cooperative Spectrum Sensing in Cognitive Radio Networks. Journal of Chinese Computer Systems. Vol. 30 (2010) No. 9, p.1658 - 1665.

[6]. Liang Yingchang, Chen Kwangcheng, Geoffrey Yeli, et al. Cognitive Radio Networking and Communications: an overview. IEEE Transactions on Vehicular Technology. Vol. 60 (2011) No. 7, p.3386-3407

[7]. Chen Xiaosi, Hang Yiling. Double-threshold Cooperative Spectrum Sensing in Small Sample Energy Detection. Computer Technology and Development. Vol. 27 (2017) No. 3, p.193-196, 200.

[8]. Chen changxing, Fu Hui, Niu Dezhi, et al. Cooperative Spectrum Sensing Algorithm Based on Double-threshold Energy Detection. Systems Engineering and Electronics. Vol. 35 (2013) No. 8, p.1742 - 1746.

[9]. O.Younis, S. Fahmy. Distributed Clustering in Ad-hoc Sensor Networks: a hybrid, energy-efficient approach. Twenty-third Annual Joint Conference of the IEEE Computer and Communications Societies. Hong Kong, China, Mar. 2004, pp: 629-640. 
[10]. Saman Atapattu, Chintha Tellambura, and Hai Jiang. Energy Detection Based Cooperative Spectrum Sensing in Cognitive Radio Networks. IEEE Transactions on Wireless Communications. Vol. 10 (2011) No. 4, p.1232 - 1241. 